\title{
Measurement of edge ion temperature in W7-X with island divertor by a retarding field analyzer probe
}

\author{
Y. Li ${ }^{1,2}$, M. Henkel ${ }^{1}$, Y. Liang ${ }^{1}$, A. Knieps ${ }^{1}$, P. Drews ${ }^{1}$, C. Killer ${ }^{3}$, D. Nicolai ${ }^{1}$, J. \\ Cosfeld $^{1}$, J. Geiger ${ }^{3}$, Y. Feng ${ }^{3}$, F. Effenberg ${ }^{4}$, D. Zhang ${ }^{3}$, P. Hacker ${ }^{3}$, D. Höschen ${ }^{1}$, \\ G. Satheeswaran ${ }^{1}$, S. Liu' ${ }^{2}$, O.Grulke ${ }^{3}$, M. Jakubowski ${ }^{3}$, S. Brezinsek ${ }^{1}$, M. Otte ${ }^{3}$, O. \\ Neubauer $^{1}$, B. Schweer ${ }^{1}$, G. S. $\mathrm{Xu}^{2}$, J. Cai ${ }^{2}$, Z. Huang ${ }^{1}$ and the W7-X team ${ }^{3}$ \\ ${ }^{1}$ Forschungszentrum Jülich GmbH, Institut für Energie- und Klimaforschung - Plasmaphysik, Partner of \\ the Trilateral Euregio Cluster (TEC), 52425 Jülich, Germany \\ ${ }^{2}$ Institute of Plasma Physics, Chinese Academy of Sciences, Hefei 230031, China \\ ${ }^{3}$ Max-Planck-Institut für Plasmaphysik, Greifswald, Germany \\ ${ }^{4}$ Department of Engineering Physics, University of Wisconsin - Madison, Madison, WI 53706, USA
}

Email: y.li@fz-juelich.de

Abstract: Knowledge of the edge ion temperature $\left(T_{i}\right)$ is important to understand the edge physics in fusion plasmas. The $\mathrm{T}_{\mathrm{i}}$ edge profile has been successfully measured at $\mathrm{W} 7-\mathrm{X}$ in an island divertor configuration using a retarding field analyzer (RFA) probe on a reciprocating manipulator. The experimental observations show that in the standard configuration, an ion temperature shoulder at the plasma boundary has been observed near the location where a sudden change of the magnetic field connection length appears in the scrape-off layer (SOL) region. The value of $T_{i}$ shoulder was observed to be dependent on the edge island structure modified by the control coils, the scrape-off layer kinetic power and the electron density. A significant reduction and flattening in the edge ion temperature profile has been also observed during the nitrogen seeding from the upper horizontal divertor port. In addition, the edge ion-to-electron temperature ratio $\tau_{i / e}$ has been calculated which gradually increases with the major radius and decreases with the normalized ion collisionality. An extended two-point model including upstream ion temperature is introduced to understand the experimental measurement.

Keywords: ion temperature, retarding field analyzer, W7-X, island configuration, MultiPurpose-Manipulator

\section{Introduction}

Understanding the edge plasma transport for controlling the fusion power exhaust requires extensive knowledges of the edge plasma parameters. The edge ion temperature profile has been massively measured in tokamaks by the retarding field analyzer (RFA), such as JET [1, 2], Tore 
Supra [3], Alcator C-mod [4], ISTTOK [5], STOR-M [6] and EAST [7]. While in stellarators there are few amount of literatures reporting the ion temperature measurement, such as LHD [8] and TJ-II [9]. To date, there are still no edge ion temperature measurements for the island configuration in W7-AS or W7-X. For this purpose, this paper reports the SOL ion temperature measurement in $\mathrm{W} 7-\mathrm{X}$.

The $\mathrm{W} 7-\mathrm{X}$ is a quasi-isodynamic stellarator with an island divertor configuration [10-12]. The magnetic field is created by a set of superconducting coils which allow to realize different magnetic configurations, including different edge island configurations. The island divertor concept uses a chain of magnetic island to form an intermediate scrape-off larger (SOL) between the confinement core and the plasma-surface interaction region. The SOL profiles of electron temperature $\left(\mathrm{T}_{\mathrm{e}}\right)$ and plasma density $\left(\mathrm{n}_{\mathrm{e}}\right)$ measured by Langmuir probes in both limiter and island divertor configurations of $\mathrm{W} 7-\mathrm{X}$ have been observed to be strongly affected by the magnetic configuration $[13,14]$.

In this paper, we present the edge ion temperature profile measured by RFA in dependence of the island configuration, heating power, and plasma density. We have looked at the so-called standard configuration with the $(\mathrm{n} / \mathrm{m})=5 / 5$-islands (edge iota $=1), \mathrm{n}$, $\mathrm{m}$ being the toroidal and poloidal mode number, respectively, and modified the island size using the additional normal conducting control coils. The dependence of the ion to electron temperature ratio on normalized ion collisionality is also presented in this paper.

A simple two-point model including the upstream ion temperature is introduced to understand the RFA measurement. This model is an extended version of the two-point model with cross-field transport [15], which simplifies the momentum loss and ignores the heat convective term and dissipative effect including radiation and neutral particles.

This paper is organized as follows: Section II introduces the RFA data interpretation and the island configuration in the W7-X stellarator. The ion temperature profile dependence on island geometry, SOL power, plasma density, and impurity seeding is discussed in Section III. The collisionality dependence of the ion to electron temperature ratio is also shown in this section. In Section IV, we introduce an extended two-point model to understand the RFA measurement.

\section{Experimental setup}

W7-X is part of new generation of optimized stellarators, aiming to demonstrate the quasi-steady state operation with plasma parameters, including ion temperatures, close to those of a future fusion power plant. It has a plasma volume $\sim 30 \mathrm{~m}^{2}$, major radius $\sim 5.5 \mathrm{~m}$ and effective minor radius $\sim 0.55 \mathrm{~m}[12,16,17]$. Kinds of quasi-isodynamic configurations could be realized by 
adjusting the coil current in the 70 superconducting coils (50 non-planar coils and 20 planar coils). In addition, the edge island geometry could also be changed by 10 control coils.

A multiple-purpose manipulator (MPM) $[18,19]$ has been developed for W7-X in 2015, which is located in the outer mid-plane with $Z=-172 \mathrm{~mm}$ and toroidal angle $\varphi=-159.26^{\circ}$. A retarding field analyzer probe has been mounted onto the MPM shaft to measure the W7-X edge plasma profiles (as seen in figure 1(c)). The RFA probe is designed based on a prototype first tested on EAST [20]. The probe is shaped to adapt to the magnetic geometry such that the entrance slits are oriented poloidally along a flux surface. The probe consists of two identical analyzers mounted back to back. For each side, the RFA components consist of a front plate with 3 entrance orifices (width $300 \mu \mathrm{m}$, length $8 \mathrm{~mm}$ per orifice), a thin plate with 3 entrance slits (width $30 \mu \mathrm{m}$, length $8 \mathrm{~mm}$ per slit and thickness $50 \mu \mathrm{m}$ ) on the front plate, three successive grids, and three collector plates. The front tungsten plate is cut with three trapezoid orifices and is $3 \mathrm{~mm}$ thick to avoid damage of the thin plate. The three slits of the thin tungsten plate are opened by laser cutting with the width to be the order of the Debye length $\left(\lambda_{D}=\sqrt{\varepsilon_{0} k_{B} T_{e} / n_{e} e^{2}}\right.$, where $\varepsilon_{0}$ is the vacuum permittivity, $k_{B}$ is the Boltzmann constant, $\mathrm{T}_{\mathrm{e}}$ and $\mathrm{n}_{\mathrm{e}}$ are the electron temperature and density, $\mathrm{e}$ is the elementary charge) to obstruct the thermal electrons. The three tungsten successive grids were cut by a square hole array with each square size $0.4 \mathrm{~mm}$ and the bar width $0.1 \mathrm{~mm}$. The three copper collector plates were fixed at a ceramic base and located parallel with the respective slits to collect the modulated current. The collector channel names are shown in figure 1(b) with 11, 12 and 13 radially arranged on the backward side and 21, 22 and 23 on the forward side. The spacer of all these sheets are $1 \mathrm{~mm}$ thick and isolated by identical ceramic sheets.

During the experiment, the slit plate potential was floated to protect its conducting wire. DC voltages are biased to the $1^{\text {st }}(-100 \mathrm{~V})$ and $3^{\text {rd }}(-200 \mathrm{~V})$ grids. Adjustable AC voltage is biased to the $2^{\text {nd }}$ grid to retard the ion flux. Three of the total six channels (No. 11, 12 and 23) have measured the modulated ion current during OP1.2b experiment, demonstrating the functionality of RFA. Due to noise contamination, in this paper, we focus on one channel (No. 23) with better signal to noise ratio to study the $T_{i}$ profile. This channel is located at the forward side since the magnetic field directs to its entrance orifice as shown in figure 1(c) where the other side is named as the backward side. Because the ion temperature upstream-downstream asymmetries increase with increasing the local plasma flow velocity $[6,21]$, the local ion temperature could only be represented by the single side measurement under a low local Mach number experiment. In W7$\mathrm{X}$, the Mach numbers in the edge island in standard configuration have been measured by a Mach probes located at the top of the IPP-FLUC1 probe. The result has been published in reference [14] and are also shown in figure 2, where "EIM+252" is naming convention for the standard 
configuration with a field strength of $2.52 \mathrm{~T}$ at phi $=0^{\circ}$ (ECRH-launching plane) at the magnetic axis. The measured Mach number is typically small $\sim 0$ in medium and particularly high plasma density experiments under the standard configuration without the control coil current. The increased control coil current would slightly increase the magnitude of Mach to $\sim 0.2$ with direction along the magnetic field, i.e., to channel 23. Therefore, it's suitable to study the edge ion temperature measured by channel 23 in the high plasma density $\left(\mathrm{n}_{\mathrm{el}}>7 \times 10^{19} \mathrm{~m}^{-3}\right)$ experiments. Note that the edge $T_{i}$ shown in this paper is measured by one side, additional $T_{i}$ error could exist if Mach number deviates from $\sim 0$.

Besides the RFA components, there are also five Langmuir tips arranged at the RFA probe top (seen in figure 1(c)). Three of the total five tips were used as a triple probe in OP1.2b to provide the plasma information of floating potential, potential measured by positive probe and ion saturated current.

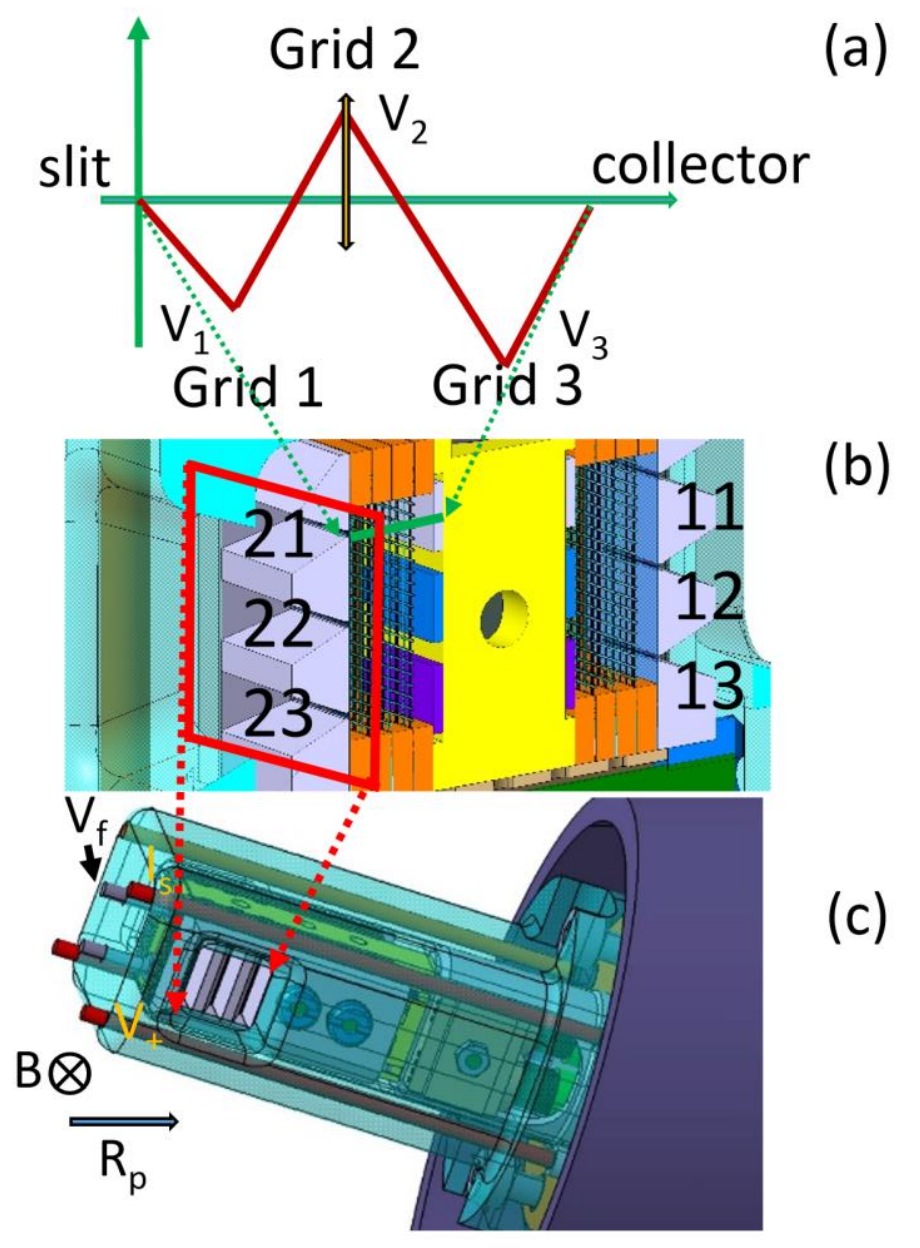

Figure 1. (a) biasing voltage scheme; (b) zoomed in RFA cavity; (c) the RFA probe head drawing. 


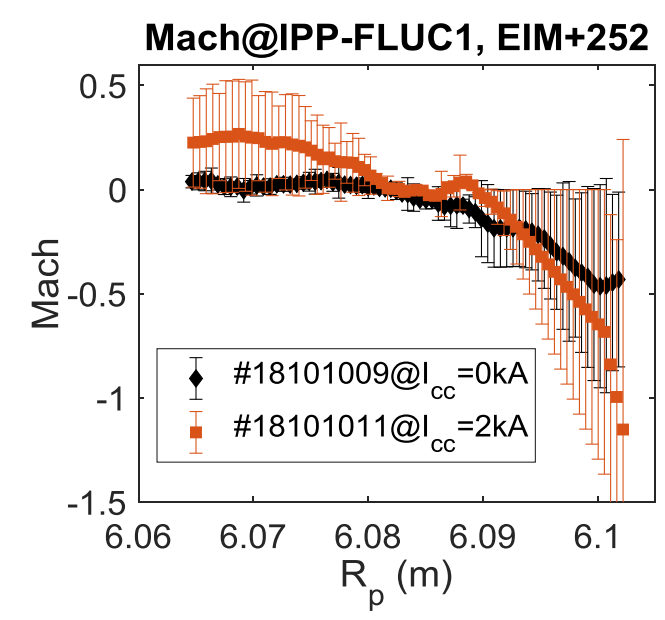

Figure 2. Mach profile measured by IPP-FLUC1 probe. These two discharges have the same ECRH heating power, $4.5 \mathrm{MW}$, and line integrated plasma density, $7 \times 10^{19} \mathrm{~m}^{-2}$. Note that the positive Mach number direction is along the magnetic field, i.e., to channel 23.

\subsection{RFA data interpretation}

The measured RFA current depends on the retarding voltage and the ion distribution function through the following formula [1] after assuming that fuel ions of charge $\mathrm{eZ}_{\mathrm{i}}$ dominate the incident flux,

$$
I_{C}=A_{\text {beam }} e Z_{i} \int_{\sqrt{2 e Z\left(V_{2}-V_{s}\right) / m_{i}}}^{\infty} \xi_{\text {total }} v_{\|} f\left(v_{\|}\right) d v_{\|}
$$

where $I_{c}$ is the RFA collector current, $A_{b e a m}$ is the beam area, $e$ is the electron charge, $m_{i}$ is the ion mass, $Z_{i}$ is the ion charge number, $V_{2}$ is the retarding sweep voltage, $V_{\mathrm{s}}$ is the sheath potential, $\xi_{\text {total }}$ is the total ion transmission coefficient, $f\left(v_{\|}\right)$is the ion distribution function for the velocities parallel to the magnetic field line. Assuming that the ion velocity distribution function in the pre-sheath edge is Maxwellian, Eq.1 can be simplified to

$$
I_{C}= \begin{cases}I_{0}, & V_{2}<V_{s} \\ I_{0} e^{-\left(V_{2}-V_{s}\right) Z / T_{i}}, & V_{2} \geq V_{s}\end{cases}
$$

where $I_{0}$ is the maximum ion current when no ions are repelled by the retarding potential $V_{2}$, and $V_{s}$ is the sheath potential. The ion temperature including fitting error is obtained by fitting the IV curve measured by RFA based on formula (2). The temporal evolution of $I_{c}$ measured by channel 23 for three discharges are shown in figure 3. These three discharges have different control coil current $\left(\mathrm{I}_{\mathrm{cc}}\right)$ setting, i.e., $\mathrm{I}_{\mathrm{cc}}=0 \mathrm{kA}, 1 \mathrm{kA}$ and $2 \mathrm{kA}$ for discharge \#180905020, $\# 180905014$ and \#180905012, respectively. The control coil effect on the ion temperature measurement will be further discussed in the follow sections. Figure 4 illustrates three individual 
RFA characteristics of channel 23, extracted at the time slice [5.250 5.252] s. The individual exponential fits according to formula (2) are also included, clearly demonstrating that the spread of parallel ion velocities may be reasonably described by a Maxwellian distribution with characteristic temperature $T_{i}$. The fluctuation of the collector current as shown in figure 4 is composed of the plasma turbulence which carry plasma information and the hardware noise. The hardware noise is mainly influenced by the pre-amplifier with noise frequency mainly $>60 \mathrm{kHz}$, where the pre-amplifier has a $-3 \mathrm{~dB}$ point of about $800 \mathrm{kHz}$.

Since the SOL plasma density could attain $\sim 10^{19} \mathrm{~m}^{-3}$, the space-charge limited current, $\mathrm{I}_{\mathrm{sc}}$, appears to be one of the biggest challenge to proper operation of the retarding field analyser. $\mathrm{I}_{\mathrm{sc}}$ could be estimated by the follow expression [22, 23]

$$
j_{s c} \approx 5.44 \times 10^{-8} \frac{\left(q V_{3}+E_{\|}\right)^{3 / 2}}{\left(z-z_{m}\right)^{2}}\left[1+0.0247 T_{i}^{1 / 2} \times\left(q V_{3}+E_{\|}\right)^{-1 / 2}\right]
$$

where $V_{3}$ is the potential applied to the third grid in volts, $E_{\|}$is the initial average ion energy in electronvolts, $\left(\mathrm{z}-\mathrm{Z}_{\mathrm{m}}\right)$ is the distance between the minimum and maximum potentials in $\mathrm{cm}$, and $\mathrm{T}_{\mathrm{i}}$ is the ion temperature in $\mathrm{eV}$. To estimate the current, which is the measured quantity, knowledge of the beam dimension is required. Based on Brillouin's theorem [24], in the presence of the magnetic field, ion beam will be confined to a constant radius without divergence up to a maximum space charge density of $n_{B}=\varepsilon_{0} B^{2} / m_{i}$. Then the beam area for hydrogen ions with an average parallel energy of $E_{\|}=100 \mathrm{eV}$ and magnetic field $\mathrm{B}=2 \mathrm{~T}$ will be limited to the geometrical slit area only if the Brillouin flux remains below a value $i_{B}=e n_{B} A_{\text {slit }} \sqrt{2 E_{\|} / m_{i}} \sim 0.1$ $\mathrm{mA}$, where $\mathrm{A}_{\text {slit }}$ is the area of the entrance slit. Since the measured RFA current is much higher than the Brillouin current, thus the ion beam radius spreads to be $2 \rho_{\mathrm{i}} \sim 1 \mathrm{~mm}$ of Larmor radius inside the RFA cavity. For $\mathrm{V}_{3}=200 \mathrm{~V}, \mathrm{~T}_{\mathrm{i}}=50 \mathrm{eV}$ and considering the dimensions of the RFA probe (grid spacing of $1 \mathrm{~mm}$, slit length of $8 \mathrm{~mm}$ ), $\mathrm{I}_{\mathrm{sc}}$ is estimated to be $\sim 2 \mathrm{~mA}$, approximate one order larger than the JET RFA's limitation [2]. 


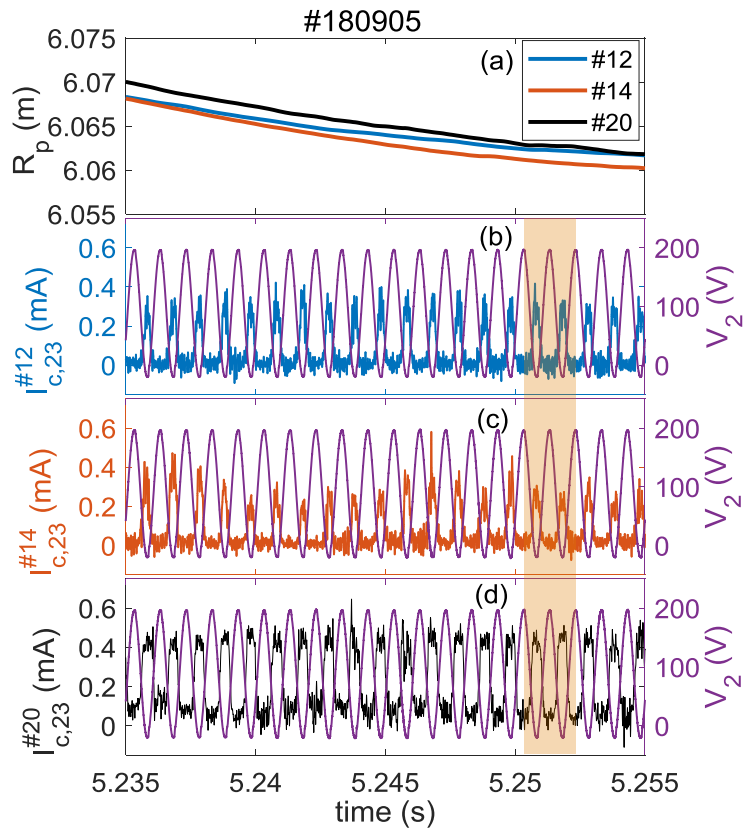

Figure 3. Temporal evolutions of the collector current of channel 23 and scanning voltage for discharges (b) \#180905012, (c) \#180905014 and (d) \#180905020, and (a) the corresponding radial position of channel 23 .

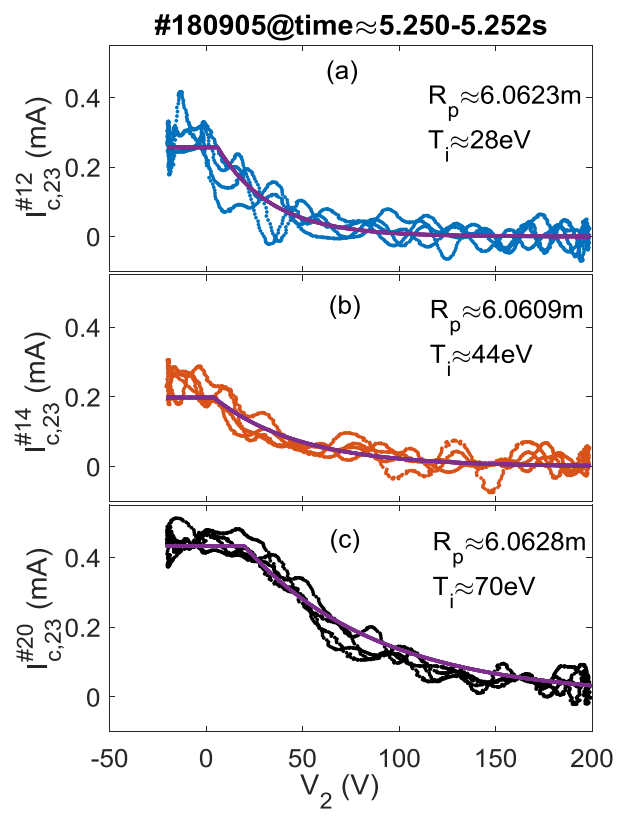

Figure 4. Example RFA characteristics at time $\approx[5.2505 .252] \mathrm{s}$. The fitted curves are obtained through using Eq. (2) and assuming $\mathrm{Z}=1$ (hydrogen plasma). The time interval corresponds to the shaded span as shown in figure 3.

\subsection{Dependence of island configuration on control coil current}

In addition to the set of superconducting coils, W7-X contains 10 normal conducting control coils behind the baffle plates which can be used to manipulate the edge islands [25]. In the experiments reported here, an identical positive dc current was applied to all 10 control coils to generate field 
components of the form $\mathrm{n} / \mathrm{m}=\left(5^{*} \mathrm{k}\right) / \mathrm{m}$ which ideally would mainly couple with $\mathrm{k}=1$ to the $5 / 5$ resonance. This results in both an increase of the island size as well as a decrease of the connection length. The Poincare plot and the connection length distribution affected by the control coils have been calculated with the field line tracing web service [26]. The magnetic field generated by 10 control coils is superimposed into the ideal standard configuration to consider the control coil effect. The magnetic connection length is calculated after considering the first wall components, including the 10 divertor plates. For simplicity, only the case without plasma current is considered. The effect of the plasma current on the edge island structure in the vicinity of the manipulator was studied in reference [27]. The preliminary result shows that increasing the plasma current at the magnetic axis increases the connection length near the remnant island region. While the changes in the plasma density and temperature profiles is still not fully explained due to lack of larger scale of plasma current scan experiments.

Figure 5 shows the $5 / 5$ islands of the standard configuration with two different control coil cases, together with the divertor and baffle plates. As can be seen in this figure, the RFA measurement path shown in the yellow-green line is much closer to the island $O$ point than to the $X$ point. An increase of $\mathrm{I}_{\mathrm{cc}}$ expands the island size and slightly shifts the island $\mathrm{O}$ point towards the divertor target. This effect changes the magnetic field connection length profile as seen in the zoomed-in figure 6. This figure shows 2D connection length plots in the R-Z plane of the MPM (color coded), a Poincare plot (red dots) and the path of the RFA (yellow line) for different control coil currents. To guide the eye, a black curve connecting to islands $\mathrm{O}$ and $\mathrm{X}$ point is added. The area of infinity connection length (marked in yellow) to the left of the edge island structure indicated by the Poincare plot is the confined area. As seen in figure 6(a), due to the island geometry, an infinity connection length region is formed at the island remnant region. With increasing the control coil current $\mathrm{I}_{\mathrm{cc}}$, the edge island is enlarged, as shown in figures 5 and 6 , and interacts more efficiently with the divertor plate. As a consequence, the $2 \mathrm{D}$ connection length distribution would be modified, including shifting the separatrix inward and shrinking the infinity connection length region near the island $\mathrm{O}$ point. We also note that in the boundary island region additional islands appear running helically around the island $\mathrm{O}$ point which are seen in figure 5 as well as in figure 6 (b) and (c). This phenomenon is caused by the control coils generating not only the $b(m, n)=5 / 5$ Fourier harmonic but also sidebands in the Fourier space. These islands can additionally contribute to the reduction of the connection length outside the confinement region (yellow region in figure 6).

To clearly show the control coil effect on the magnetic connection length, profiles along the RFA probe path and island $\mathrm{O}$ to $\mathrm{X}$ point path are plotted in figure 7 (a) and (b). The connection length gradually decreases with the major radius from the separatrix and then is approximately constant 
in the range of [6.05 6.08] $\mathrm{m}$, across a large part of the island. A steep drop of the connection length profile can be observed at $\mathrm{R} \approx 6.08 \mathrm{~m}$ where the value changed from $\sim 300 \mathrm{~m}$ to $\sim 20 \mathrm{~m}$. An increase of the control coil current from $\mathrm{I}_{\mathrm{cc}}=0 \mathrm{kA}$ to $2 \mathrm{kA}$, causes the connection length in the intermediate region to decrease by $30 \%$ and the LCFS to shift inwards by $\sim 1 \mathrm{~cm}$. The control coils also cause the connection length in the edge island remnant to strongly decrease and effectively disappear, which is reflected in figure 6 and $7(\mathrm{~b})$.

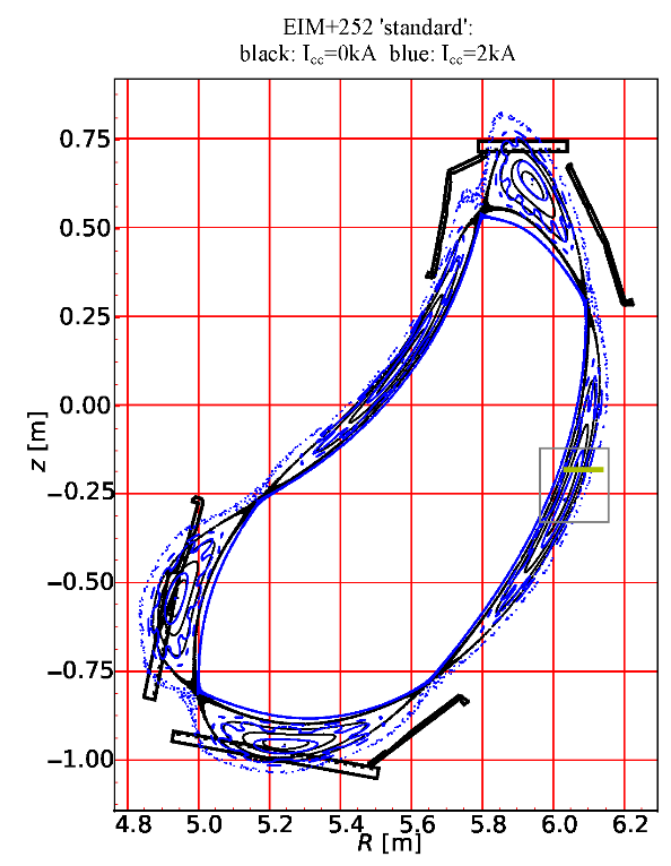

Figure 5. Poincaré plots of standard configuration with different control coil cases: (black) $\mathrm{I}_{\mathrm{cc}}=0 \mathrm{kA}$; (blue) $\mathrm{I}_{\mathrm{cc}}=2 \mathrm{kA}$. The yellow-green line is the RFA channel 23 measurement path. Note that these results are obtained in vacuum approximation.
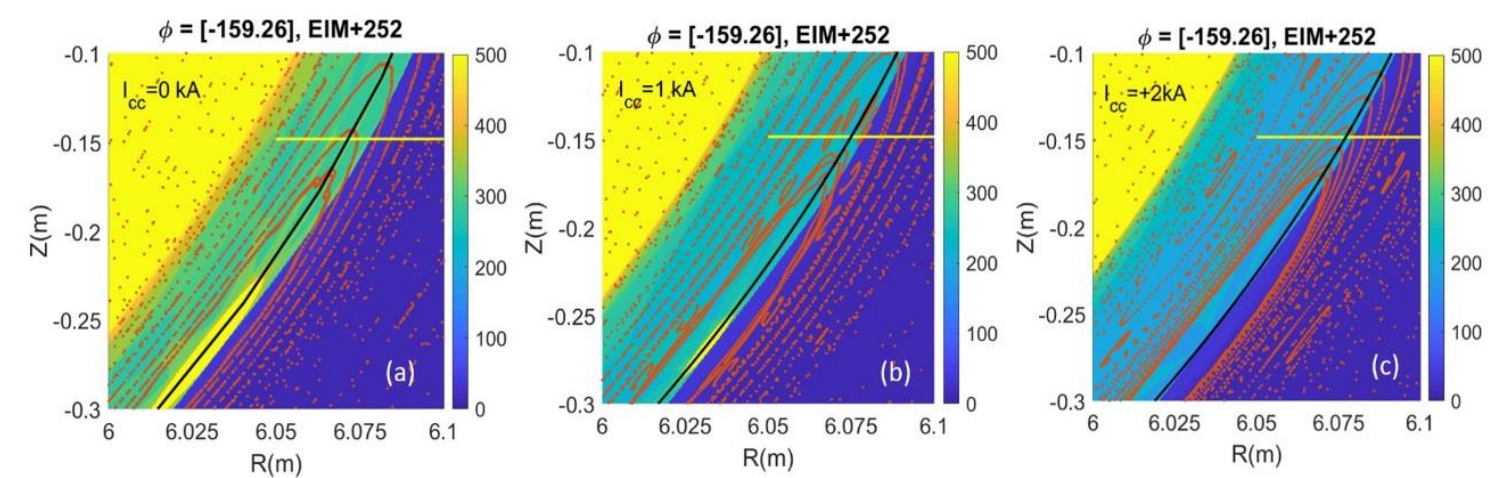

Figure 6. Connection lengths profiles and superimposed standard configuration Poincaré plots of different control coil cases: (a) $I_{c c}=0 \mathrm{kA}$; (b) $I_{c c}=1 \mathrm{kA}$; and (c) $I_{c c}=2 \mathrm{kA}$. The yellow line is the RFA channel 23 measurement path. The black curve connects the island $\mathrm{O}$ point and $\mathrm{X}$ point. Note that these results are obtained in vacuum approximation. 

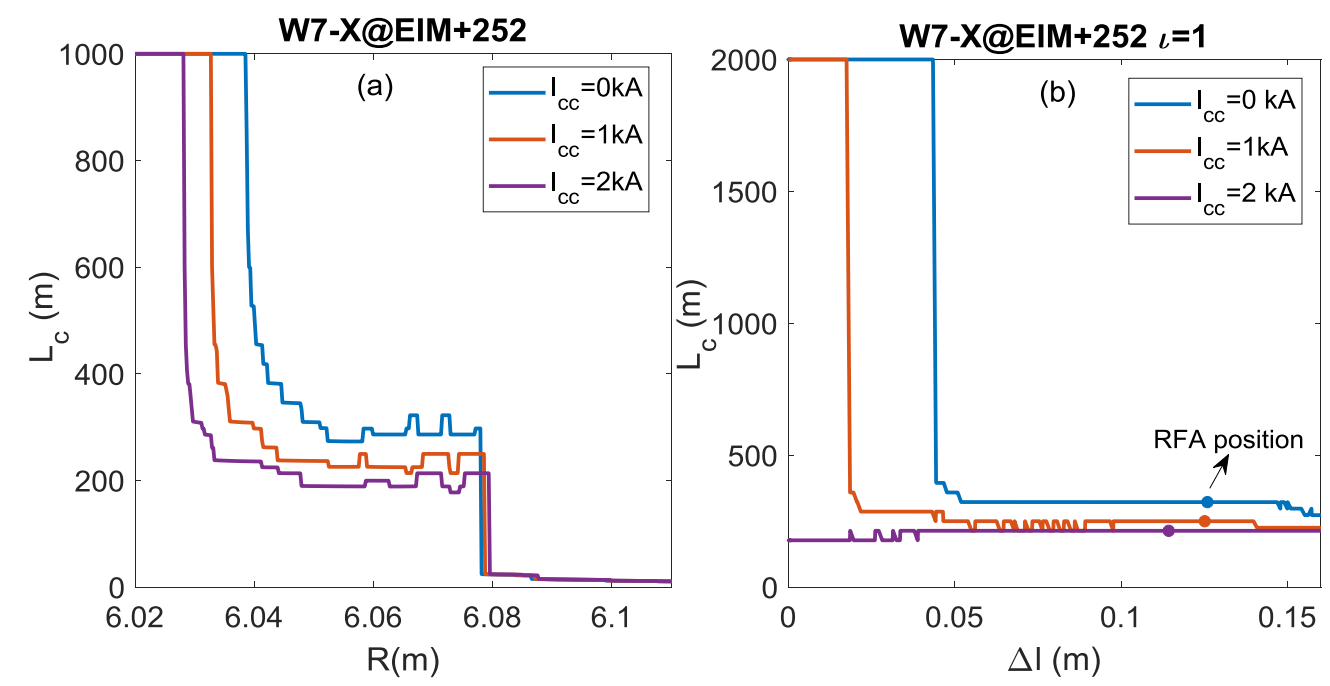

Figure 7. (a) radial profiles of connection length along the yellow line as shown in figure 6 for different $\mathrm{I}_{\mathrm{cc}}$ values; (b) connection length profile along the black curve as shown in figure 6 for different $\mathrm{I}_{\mathrm{cc}}$ values. $\Delta \mathrm{l}$ is the arc length starting from the island $\mathrm{O}$ point. The solid point is corresponding to the intersection of the yellow line and black curve. Note that these results are obtained in vacuum approximation.

\section{Edge ion temperature and ion to electron temperature evolution in standard configuration}

The RFA experiments are only carried out in the ECRH heating plasma with gas puffing to control the plasma density. In this case, the ion energy is mainly transferred from the electron energy by the ion to electron collisions. And the plasma density at separatrix could be assumed to be proportional to the central line averaged density. Since the SOL radiation would also influence the edge $T_{i}$ for a fixed perpendicular heat flux through the separatrix, the SOL kinetic power PSOL estimated by formula $\mathrm{P}_{\mathrm{SOL}}=\mathrm{P}_{\mathrm{ECRH}}-\mathrm{P}_{\mathrm{rad}}$ is introduced to study its dependence of the $\mathrm{T}_{\mathrm{i}}$ shoulder. The bolometer system in W7-X [28] has been used to monitor the total radiative power loss, $\mathrm{P}_{\mathrm{rad}}$, covering the core and SOL region. The edge ion temperature and ion to electron temperature ratio have been studied by slightly varying the edge island size by turning the control coil current $\mathrm{I}_{\mathrm{cc}}$, $\mathrm{P}_{\mathrm{SOL}}$ and plasma density in the standard configuration. The effect of impurity seeding is also introduced in this section. The respective effect on the ion temperature profile would be discussed in detail in the following subsection.

\subsection{Dependence of edge ion temperature on the control coil current}

The ion temperature profiles for six discharges with a constant ECRH power $\mathrm{P}_{\mathrm{ECRH}}=3 \mathrm{MW}$ and a central line integrated plasma density of $\mathrm{n}_{\mathrm{el}}=7 \times 10^{19} \mathrm{~m}^{-2}$, except $\mathrm{n}_{\mathrm{el}}=8 \times 10^{19} \mathrm{~m}^{-2}$ in discharge $\# 014$ are presented in figure $8 . \mathrm{P}_{\mathrm{SOL}}$ in these selected experiments are roughly constant, $2.5 \mathrm{MW}$. In these experiments, positive DC current was applied to the 10 control coils to expand the 5/5 island geometry. In this figure, three discharges \#018, \#019 and \#020 with $\mathrm{I}_{\mathrm{cc}}=0 \mathrm{kA}$ show similar $\mathrm{T}_{\mathrm{i}}$ 
profiles. An ion temperature shoulder is formed with the shoulder edge located radially at $\sim 6.08$ $\mathrm{m}$ which is near the steep drop position of the magnetic connection length profile. The flattening of the ion temperature profiles coincides with the constant connection length in the intermediate region. This result indicates that the observed ion temperature shoulder structure may be attributed to the island configuration. When $\mathrm{I}_{\mathrm{cc}}$ gradually increases from $0 \mathrm{kA}$ to $2.5 \mathrm{kA}$, the ion temperature drops by almost $50 \%$. A relation between the $T_{i}$ shoulder and $I_{c c}$ is shown in figure 9 , where the $\mathrm{T}_{\mathrm{i}}$ shoulder value is averaged over a window of $1 \mathrm{~cm}, \mathrm{R}_{\mathrm{p}} \approx[6.066 .07] \mathrm{m}, 2-3 \mathrm{~cm}$ away from the separatrix. The error of the $T_{i}$ shoulder is the upper and lower limits of $T_{i}$ in the selected window. As seen in figure 9, a tendency towards lower temperatures for higher control coil currents is visible. While it should be emphasized that enlarged island size not only reduces the connection length but also shifts inward the separatrix. The latter could also reduce the ion temperature. Note that in the far SOL region $\mathrm{R}_{\mathrm{p}}>6.09 \mathrm{~m}$, the RFA measurement is less accurate due to larger noise induced by small modulated ion current amplitude.

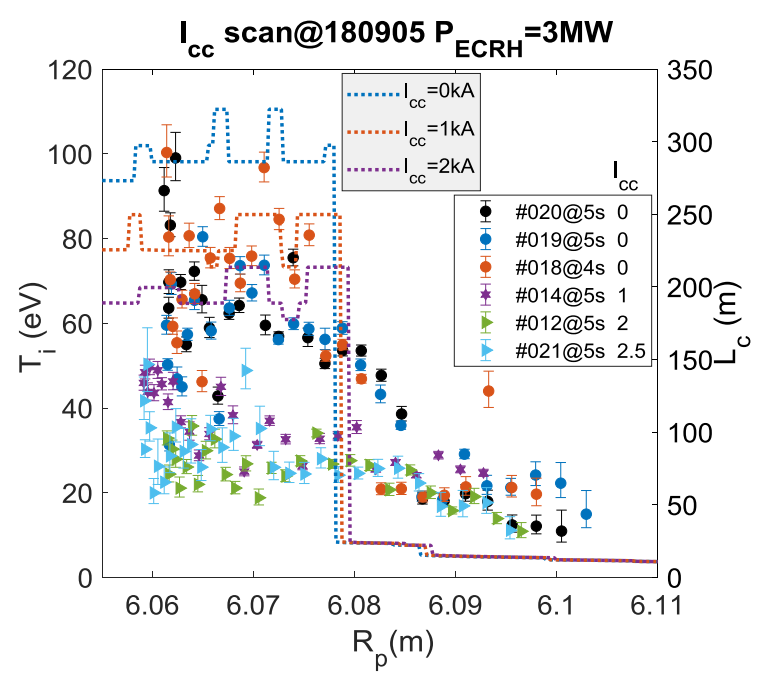

Figure 8. Dependence of edge ion temperature profiles on control coil current. The covered dash lines are the connection length profiles for different control coil cases.

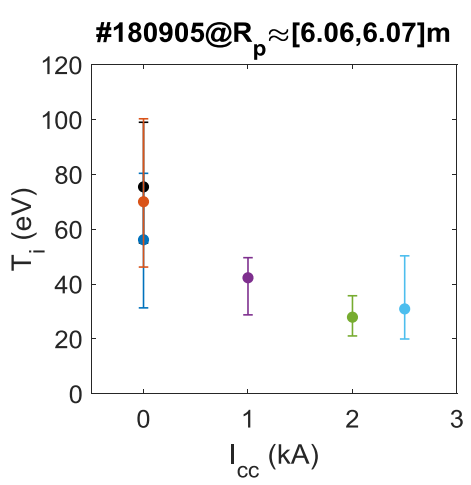

Figure 9. Edge $T_{i}$ over a window $R_{p} \approx[6.066 .07] \mathrm{m}$ or $\Delta r \approx[0.020 .03] \mathrm{m}$ as a function of $\mathrm{I}_{\mathrm{cc}}$, where $\Delta \mathrm{r}$ is the distance between the RFA measurement position and the separatrix. Note that the dot color is corresponding to figure 8 . 


\subsection{Dependence of edge ion temperature on PsoL}

The edge ion temperature profile has been studied in three ECRH heating power steps (2MW, $3 \mathrm{MW}$, and $5 \mathrm{MW}$ ) under different $\mathrm{I}_{\mathrm{cc}}$ conditions for fixed $\mathrm{n}_{\mathrm{el}}$. These $\mathrm{T}_{\mathrm{i}}$ profiles measured from 7 discharges with additional control coil operation are illustrated in figure 10. The result shows that the ion temperature shoulder increases with the ECRH heating power for fixed $\mathrm{I}_{\mathrm{cc}}$ and $\mathrm{n}_{\mathrm{el}}$. In the case of $\mathrm{I}_{\mathrm{cc}}=0$, the $\mathrm{T}_{\mathrm{i}}$ shoulder increases from $\sim 40 \mathrm{eV}$ (\#027) to $\sim 60 \mathrm{eV}$ (\#020) as the heating power increases from $2 \mathrm{MW}$ to $3 \mathrm{MW}$. In the case of $\mathrm{I}_{\mathrm{cc}}=+1 \mathrm{kA}$, the $\mathrm{T}_{\mathrm{i}}$ shoulder, as shown in hexagonal star dots, increases by a factor of $\sim 3$ as $\mathrm{P}_{\mathrm{ECRH}}$ increases from $3 \mathrm{MW}$ to $5 \mathrm{MW}$. In the case of Icc $=+2 \mathrm{kA}$, the $T_{i}$ shoulder increases by a factor of $\sim 2$ as $P_{E C R H}$ increases from $3 \mathrm{MW}$ to $5 \mathrm{MW}$. The control coil current has also been found to reduce the $T_{i}$ shoulder. Comparing with the case of $I_{c c}=0$, the $T_{i}$ shoulder has been reduced when $I_{c c}$ was increased to $1 \mathrm{kA}$ and $2 \mathrm{kA}$. The increasing tendency of the $T_{i}$ shoulder as a function of $P_{E C R H}$ is probably caused by increased heat flux through the separatrix as the ECRH power increases. To eliminate the radiation effect, the $T_{i}$ shoulder as a function of $\mathrm{P}_{\mathrm{SOL}}$ is shown in figure 11. The $\mathrm{T}_{\mathrm{i}}$ shoulder is observed to gradually increase with $\mathrm{P}_{\mathrm{SOL}}$ for certain control coil current. For $\mathrm{I}_{c c}=0$, the dependence of $\mathrm{T}_{\mathrm{i}}$ from $\mathrm{P}_{\mathrm{SOL}}$ is stronger than in the case of $I_{c c}=1 \mathrm{kA}$ or $2 \mathrm{kA}$. Note that the radiation distribution in SOL should also affect the edge ion temperature profile, especially the radiative power distribution between the upstream and downstream plasma region, which cannot be estimated from the bolometer system in its current status.

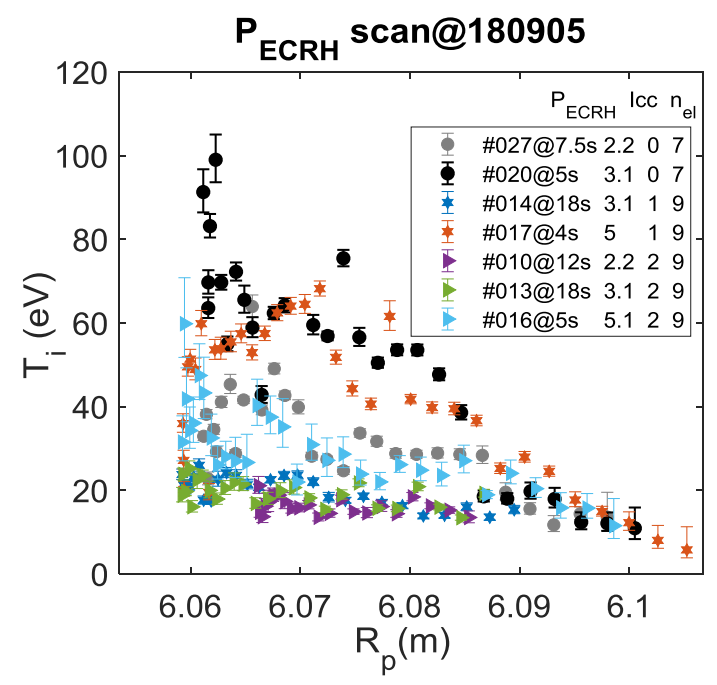

Figure 10. Dependence of edge ion temperature profiles on $P_{E C R H}$ for different control coil cases. Note that the line integrated plasma density $\mathrm{n}_{\mathrm{el}}$ is in $10^{19} \mathrm{~m}^{-2}$. 


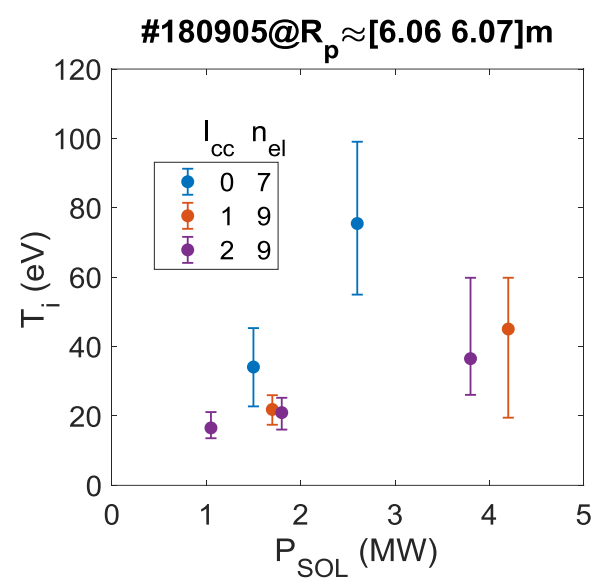

Figure 11. Edge $T_{i}$ as a function of $P_{S O L}$. Note that the line integrated plasma density $n_{\mathrm{el}}$ is in $10^{19} \mathrm{~m}^{-2}$.

\subsection{Dependence of edge ion temperature on $\mathbf{n}_{\mathrm{el}}$}

Since the RFA measurements were limited to high density plasmas, the dependence of the $T_{i}$ shoulder on the plasma density is studied in high density scan under different $\mathrm{I}_{\mathrm{cc}}$ and fixed $\mathrm{P}_{\mathrm{ECRH}}=3$ MW. As can be seen in figure 12, for the case of $\mathrm{I}_{\mathrm{cc}}=0 \mathrm{kA}$, the $\mathrm{T}_{\mathrm{i}}$ shoulder decreases from $\sim 60 \mathrm{eV}$ in discharge $\# 020\left(\mathrm{n}_{\mathrm{el}}=7 \times 10^{19} \mathrm{~m}^{-2}\right)$ to $40 \mathrm{eV}$ in $\# 015\left(\mathrm{n}_{\mathrm{el}}=9 \times 10^{19} \mathrm{~m}^{-2}\right)$. For the case of $\mathrm{I}_{\mathrm{cc}}=1 \mathrm{kA}$, the $\mathrm{T}_{\mathrm{i}}$ shoulder gradually decreases from $\sim 40 \mathrm{eV}$ in $\# 014\left(\mathrm{n}_{\mathrm{el}}=8 \times 10^{19} \mathrm{~m}^{-2}\right)$ to $\sim 25 \mathrm{eV}$ in \#014 $\left(\mathrm{n}_{\mathrm{el}}=9 \times 10^{19} \mathrm{~m}^{-2}\right)$. For the case of $\mathrm{I}_{\mathrm{cc}}=2 \mathrm{kA}$, the $\mathrm{T}_{\mathrm{i}}$ shoulder gradually decreases from $\sim 30 \mathrm{eV}$ in $\# 021\left(\mathrm{n}_{\mathrm{el}}=7 \times 10^{19} \mathrm{~m}^{-2}\right)$ to $\sim 20 \mathrm{eV}$ in \#013 $\left(\mathrm{n}_{\mathrm{el}}=9 \times 10^{19} \mathrm{~m}^{-2}\right)$. A much clearer dependence of the $\mathrm{T}_{\mathrm{i}}$ shoulder on the $\mathrm{n}_{\mathrm{el}}$ is shown in figure 13. The result shows that in the high density regime, the ion temperature gradually decreases with increasing the plasma electron density. Since the bolometry system has observed that the plasma radiation increase as the plasma density increase, $\mathrm{P}_{\mathrm{SOL}}$ has been reduced by $12 \%$ for Icc $=0 \mathrm{kA}, 26 \%$ for $I c c=1 \mathrm{kA}$ and $22 \%$ for $I c c=2 \mathrm{kA}$. Then the $\mathrm{n}_{\mathrm{el}}$ dependence of the $T_{i}$ shoulder is coupled with the $P_{S O L}$ dependence, which also reduce the $T_{i}$ shoulder as the plasma density increases. Combining the observation of the $T_{i}$ shoulder dependence on $\mathrm{P}_{\mathrm{SOL}}$ and $\mathrm{n}_{\mathrm{el}}$, it's reasonable to speculate that the $\mathrm{T}_{\mathrm{i}}$ shoulder decreases with the collisionality, which will be further studied in section 3.5. 


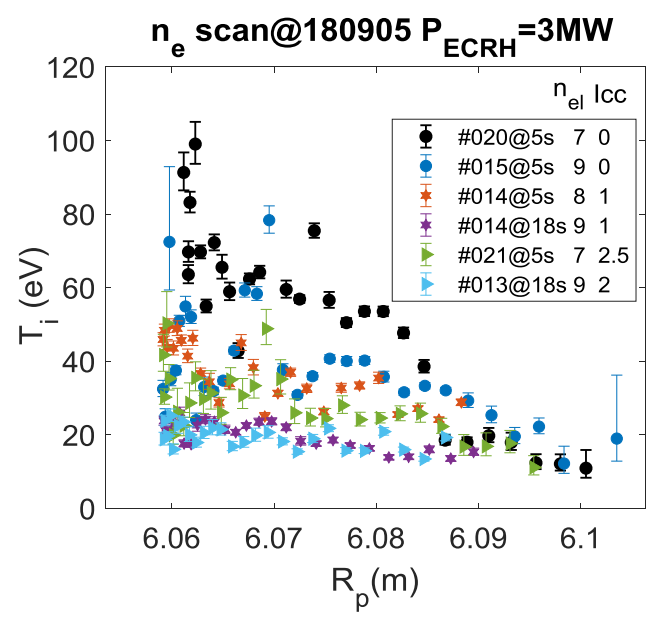

Figure 12. Dependence of edge ion temperature profiles on $n_{\mathrm{el}}$ for different control coil values. Note that the line integrated plasma density $\mathrm{n}_{\mathrm{el}}$ is in $10^{19} \mathrm{~m}^{-2}$.

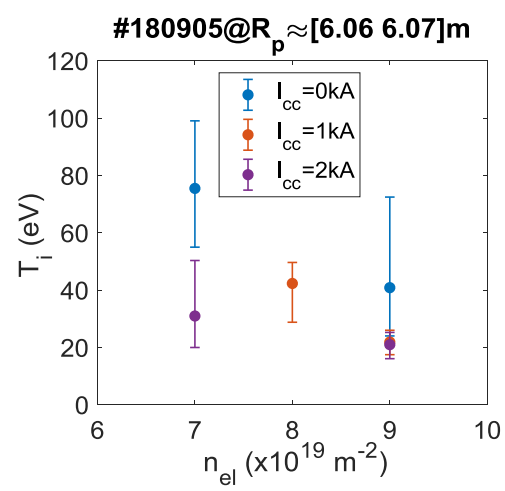

Figure 13. Edge $T_{i}$ as a function of $n_{e l}$.

\subsection{Impurity seeding effect on the ion temperature profiles}

In some experiments covered by the RFA probe, nitrogen was injected close to the divertor in a divertor module that is directly magnetically connected to the MPM, i.e. shares the same island with a distance of about $7 \mathrm{~m}$. Upstream ion temperature located near the MPM measurement position is observed to be reduced by the downstream nitrogen seeding. The amounts of injected nitrogen are $\sim 5 \times 10^{19}$ particles per puff with puffing rate $5 \times 10^{20}$ atoms/s. In discharge \#027 $\left(\mathrm{P}_{\mathrm{ECRH}}=2.2 \mathrm{MW}, \mathrm{n}_{\mathrm{el}}=7 \times 10^{19} \mathrm{~m}^{-2}\right), \mathrm{RFA}$ was plunged twice before $(7.5 \mathrm{~s})$ and after $(9 \mathrm{~s})$ the nitrogen seeding injection (8s). The measurements with the RFA show in figure 14 that the ion temperature has been reduced by about $50 \%$ due to nitrogen seeding. The tendency of the $\mathrm{T}_{\mathrm{i}}$ profile is also reversed by the nitrogen impurities, i.e., $\mathrm{T}_{\mathrm{i}}$ gradually increases with the major radius in the radial position $\sim$ [6.06 6.078] $\mathrm{m}$ in discharge \#027 at 9s. In addition, the edge ion temperature has been observed to be flattened by the nitrogen injection. In discharge \#024 ( $\mathrm{P}_{\mathrm{ECRH}}=3.1 \mathrm{MW}$, $\left.\mathrm{n}_{\mathrm{el}}=7 \times 10^{19} \mathrm{~m}^{-2}\right)$ which is similar to discharge \#020, the RFA has been plunged twice $(7.5 \mathrm{~s}$ and $9 \mathrm{~s})$ before and after the $2^{\text {nd }}$ nitrogen seeding $(8 \mathrm{~s})$. The first nitrogen seeding time is $6.2 \mathrm{~s}$. The $\mathrm{T}_{\mathrm{i}}$ profile measured in \#020 has been referred to compare with the $T_{i}$ profile measured in \#024. Compared 
with the reference $T_{i}$ profile without nitrogen seeding, the ion temperature after $1^{\text {st }}$ nitrogen seeding slightly decreases at the shoulder while increases outside the slope pedestal region which leads to flattening the shoulder structure. For the $2^{\text {nd }}$ nitrogen injection, the ion temperature is much reduced at the shoulder and the pedestal-like structure is almost disappeared.

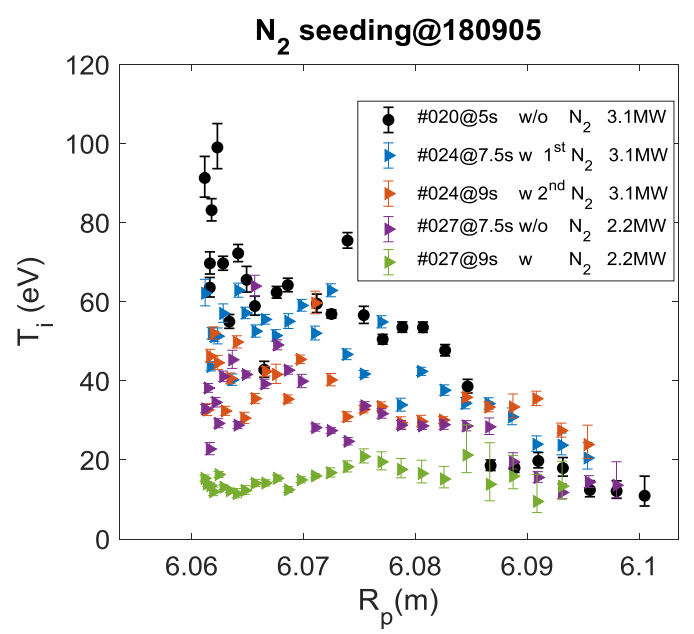

Figure 14. ion temperature affected by nitrogen seeding for the case of $\mathrm{I}_{\mathrm{cc}}=0 \mathrm{kA}$.

\subsection{Collisionality dependence of edge ion temperature and ion to electron temperature ratio}

Besides the RFA components, there are three cylindrical Langmuir tips at the top of the RFA probe to measure the floating potential, potential of the positive electrode and the ion saturated current. The two entrance plates electrically conducted with each other with each size $\sim 1 \mathrm{~cm}^{2}$ are also used to measure the floating potential. Since the distance in the poloidal direction from the positive tip to the floating tip is much larger $(12.6 \mathrm{~mm})$ than to the entrance plate centre $(8.6 \mathrm{~mm})$, to avoid the poloidal asymmetry effect caused by the island geometry, the floating potential tip is replaced by the entrance plates to calculate the electron temperature, $T_{e}=\left(V_{+}-V_{f}\right) / \ln 2$. Then the ion to electron temperature ratio is obtained. Figure 15 shows a typical set of plasma edge profiles measured by RFA. In this discharge \#180905016, the injected ECRH heating power is $5 \mathrm{MW}$ and the central line integrated plasma density is $9 \times 10^{19} \mathrm{~m}^{-2}$. The edge island configuration has been expanded by the control coils $\left(\mathrm{I}_{\mathrm{cc}}=2 \mathrm{kA}\right)$. As shown in figure 15, the plasma parameter profiles $\left(\mathrm{T}_{\mathrm{i}}, \mathrm{T}_{\mathrm{e}}\right.$ and $\left.\mathrm{n}_{\mathrm{e}}\right)$ approximately decrease with the major radius and are flat in the intermediate connection length region. The ion to electron temperature ratio $\tau_{i / e}$ profile shown in figure $15(\mathrm{~b})$ gradually increases with the major radius, which is similar with the tokamak case [29]. While the island geometry seems flattening the $\tau_{i / e}$ profile since the $\tau_{i / e}$ increment inside the intermediate region is much small than the one in the short connection length $\left(\mathrm{L}_{\mathrm{c}} \sim 10 \mathrm{~m}\right)$ region. The normalized ion collisionality in SOL $v_{S O L, i}^{*} \equiv L_{c} / \lambda_{i i} \approx 10^{-16} n_{u} L_{c} / T_{i u}^{2}$ [30] profile shown in figure $15(\mathrm{~d})$ is 
roughly varied between 20 and 100 . This $v_{S O L, i}^{*}$ value indicates that the SOL plasma transport is within the conduction-limited regime for the tokamak case [27].

To study the dependence of $\tau_{i / e}$ on $\mathrm{I}_{\mathrm{cc}}, \mathrm{P}_{\mathrm{ECRH}}$ and $\mathrm{n}_{\mathrm{el}}, \tau_{i / e}$ values are taken from the range $\mathrm{R}_{\mathrm{p}}=[6.06$ 6.07] $\mathrm{m}$ from different plasma program and are presented in figure 16 . As shown in figure $16, \tau_{i / e}$ is roughly decreased with increasing the $\mathrm{I}_{\mathrm{cc}}$ and $\mathrm{n}_{\mathrm{el}}$, while increases with increasing the $\mathrm{P}_{\mathrm{SOL}}$. This implies that $\tau_{i / e}$ would decrease with the plasma collisionality. Note that the purple dot in figure 16(b) for the case of $\mathrm{P}_{\mathrm{SOL}} \approx 1 \mathrm{MW}$ is from discharge \#180905010, where the RFA was plunged much shallower, deepest position near $\mathrm{R} \sim 6.07 \mathrm{~m}$, comparing with the other discharges. This may be the reason that the selected $\tau_{i / e}$ is much higher than the ones in the other purple plotted discharges. Except for this discharge, $\tau_{i / e}$ gradually increases with $\mathrm{P}_{\mathrm{SOL}}$ for a given control coil current.

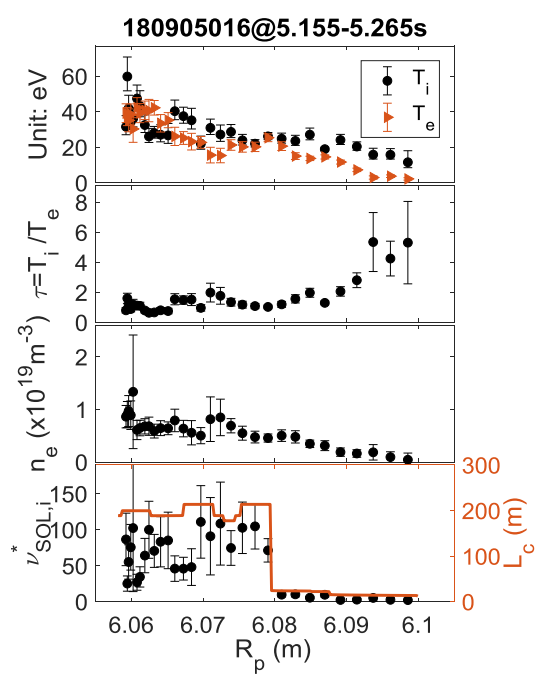

Figure 15. Radial profiles of (a) $\mathrm{T}_{\mathrm{i}}$ and $\mathrm{T}_{\mathrm{e}}$; (b) ion to electron temperature ratio $\tau_{i / e}$; (c) $\mathrm{n}_{\mathrm{e}}$ and (d) normalized ion collisionality in SOL $v_{S O L, i}^{*}$ for discharge \#180905016 with covered $\mathrm{L}_{\mathrm{c}}$ profile for $\mathrm{I}_{\mathrm{cc}}=2 \mathrm{kA}$.
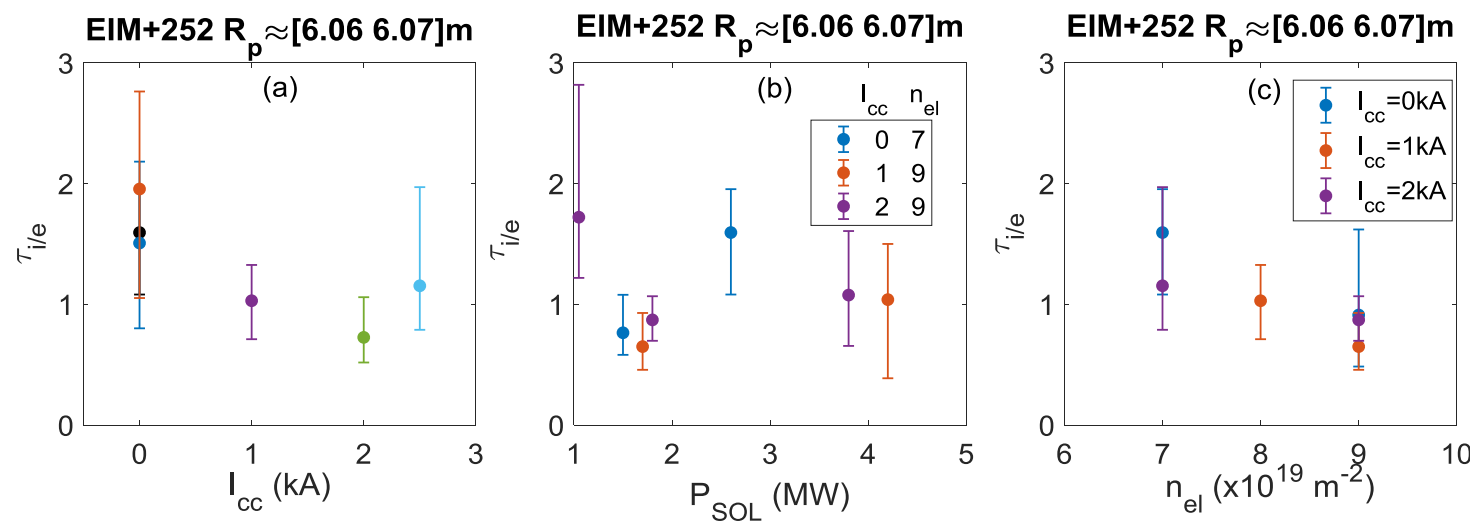
Figure 16. Ion to electron temperature ratio as a function of (a) control coil current, $\mathrm{I}_{\mathrm{cc}}$; (b) SOL power, $\mathrm{P}_{\mathrm{SOL}}$; (c) central line integrated density $\mathrm{n}_{\mathrm{el}}$. Note that the dot color in sub-figure (a) is corresponding to figure 8 .

Since the above plasma parameter dependence of $\mathrm{T}_{\mathrm{i}}$ and $\tau_{i / e}$ imply that the collisionality is probably correlated with the ion temperature, the collisionality dependence of ion temperature and ion to electron temperature is studied. In figure 17 , the plasma parameters $\mathrm{T}_{\mathrm{i}}, \mathrm{T}_{\mathrm{e}}, \mathrm{n}_{\mathrm{e}}$ and $\tau_{i / e}$ measured by the RFA probe are plotted as a function of the normalized ion collisionality $v_{S O L, i}^{*}$. As can be seen, the ion temperature gradually decreases with $v_{S O L, i}^{*}$, while the electron temperature and density are dispersed and seem independent on $v_{S O L, i}^{*}$. Increasing the control coil currents would expand the island geometry and is therefore expected to overall reduce the ion temperature, electron temperature and density. $\tau_{i / e}$ is observed to be gradually decreased with $v_{S O L, i}^{*}$ from $\sim 2$ to $\sim 1$ when the normalized ion collisionality increases from $\sim 10$ to $\sim 200$. This evolution seems independent on different control coil current conditions even though the parameters $\left(T_{i}, T_{e}, n_{e}\right.$ and $\left.L_{c}\right)$ has been changed by expanding the island geometry. This may imply that slightly tailoring the island configuration would not significantly change the ion and electron energy balance. The relation of $\tau_{i / e}$ with $v_{S O L, i}^{*}$ for the measurement region approximately follows the formula $\tau_{i / e}=8 \times v_{S O L, i}^{*-0.5}$ which is plotted as a solid red line in figure $17(\mathrm{~d})$ to aid the eye. This trend is similar to the simulation results for tokamak configuration using the EDGE2D code and an 'onion-skin method' as shown in chapter 4 of the book [30]. Comparing with the tokamak case, $\tau_{i / e}$ obtained by the RFA experiment in Tore Supra plasma with Ohmic heating indicates that $\tau_{i / e}$ first increases with $v_{S O L}^{*}$, and then gradually decreases with $v_{S O L}^{*}$ [29]. This may imply that the dependence of $\tau_{i / e}$ on $v_{S O L, i}^{*}$ in stellarator may be also not monotonically decreasing, but could increase in low $v_{S O L, i}^{*}$ region of [1 10]. 


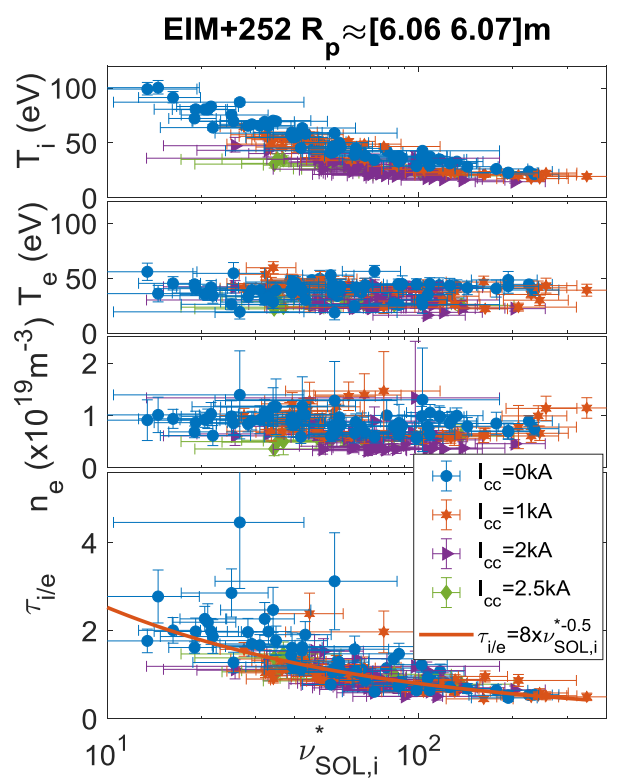

Figure 17. RFA measurement for (a) $\mathrm{T}_{\mathrm{i}}$, (b) $\mathrm{T}_{\mathrm{e}}$, (c) $\mathrm{n}_{\mathrm{e}}$ and (d) $\tau_{i / e}$ as a function of the normalized ion collisionality $v_{S O L, i}^{*}$. The solid line obeying the formula $\tau_{i / e}=8 \times v_{S O L, i}^{*-0.5}$ is used to aid the eye.

\section{Theory analysis}

A two-point model including ion temperature is introduced to calculate the upstream ion temperature $\mathrm{T}_{\mathrm{iu}}$ and electron temperature $\mathrm{T}_{\mathrm{eu}}$, and downstream electron temperature $\mathrm{T}_{\mathrm{ed}}$ and density $\mathrm{n}_{\text {ed. }}$ This model is based on Feng's two-point model including cross-field transport [15] and is extended to consider the ion energy balance equation and thermal equilibration collision term $\mathrm{Q}_{\text {eq. }}$. The heat convective transport and neutral effect on the plasma are not considered in this model. The extended two-point model equations are derived in eqs. (4.1-4.5)

$$
\begin{gathered}
T_{e u}^{\frac{7}{2}}=T_{e d}^{\frac{7}{2}}+\frac{7 q_{\| e^{e} L_{c}}}{2 \kappa_{e}}-\frac{7 Q_{e q u} L_{c}^{2}}{4 \kappa_{e}}-\frac{7 e \chi_{e}\left(n_{u}+n_{e d}\right)}{\kappa_{e} \Theta^{2}}\left(T_{e u}-T_{e d}\right) \\
T_{i u}^{\frac{7}{2}}=T_{i d}^{\frac{7}{2}}+\frac{7 q_{\| i} L_{c}}{2 \kappa_{i}}+\frac{7 Q_{e q u} L_{c}^{2}}{4 \kappa_{i}}-\frac{7 e \chi_{i}\left(n_{u}+n_{e d}\right)}{\kappa_{i} \Theta^{2}}\left(T_{i u}-T_{e d}\right) \\
n_{u}\left(T_{e u}+T_{i u}\right)=\left(1+f_{m}\right) 4 n_{e d} T_{e d} \\
q_{\| e}=n_{e d} c_{s d} \gamma_{e} T_{e d} \\
q_{\| i}=n_{e d} c_{s d} \gamma_{i} T_{e d}
\end{gathered}
$$

where $\Theta$ is the field line pitch angle, $c_{s d}=\sqrt{2 k T_{e d} / m_{i}}$. The electron and ion sheath heat transmission coefficients are $\gamma_{e}=5.3, \gamma_{i}=2.5$, respectively. The electron and ion conductivity coefficients are $\kappa_{e}=2000, \kappa_{i}=60$, respectively. $f_{m}=\frac{D}{2 \Theta c_{s d}}\left(\frac{1}{\lambda_{\Gamma}}+\int \frac{n_{e} V_{\|} / n_{d} c_{s d}}{(\Delta y / 2)^{2}} d x\right), \mathrm{D}$ is the particle diffusion coefficient, $V_{\|}$is the parallel flow, $\lambda_{\Gamma}$ is the parallel particle flux radial decay length and is set to be $0.01 \mathrm{~m}$, and $\Delta y$ is the island poloidal width, about $0.6 \mathrm{~m}$ for W7-X. The 
thermal equilibration collisions between electrons and ions satisfy $Q_{e q}=\frac{3 m_{e}}{m_{i}} n_{e} v_{e q}\left(k T_{e}-k T_{i}\right)$, and $v_{e q} \approx 2.9 \times 10^{-12} n_{e} \ln \Lambda T_{e}^{-\frac{3}{2}}$. The parallel electron heat flux $q_{\| e}=\mathrm{P}_{\mathrm{SOL}} /\left(4 \pi^{2} \mathrm{aR} \Theta\right)$, where $\mathrm{P}_{\mathrm{SOL}}$ is the total power entering the SOL, and for W7-X, $\mathrm{a}=0.52 \mathrm{~m}, \mathrm{R}=5.5 \mathrm{~m}$ and $\Theta=0.001$. The ion temperature at downstream $\mathrm{T}_{\text {id }}$ is assumed to be equal with the electron temperature at downstream $\mathrm{T}_{\mathrm{ed}}$.
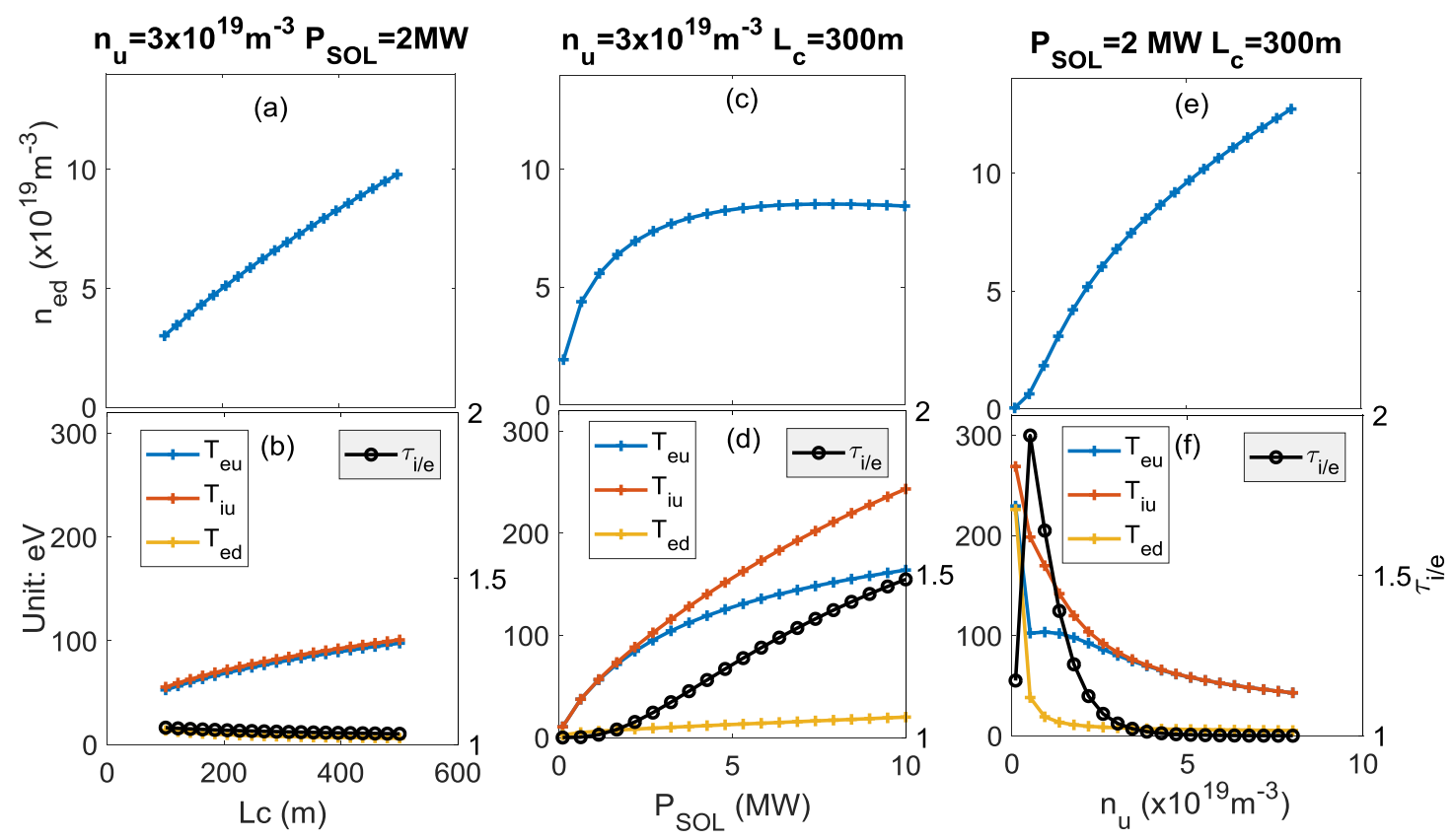

Figure 18. Two-point model: downstream electron density, $\mathrm{n}_{\mathrm{ed}}$, as a function of (a) connection length, $\mathrm{L}_{\mathrm{c}}$, (c) SOL power, $\mathrm{P}_{\mathrm{SOL}}$, and (e) upstream electron density, $\mathrm{n}_{\mathrm{u}}$; upstream ion and electron temperature, $\mathrm{T}_{\mathrm{iu}}$ and $\mathrm{T}_{\mathrm{eu}}$, downstream electron temperature, $\mathrm{T}_{\mathrm{ed}}$, and upstream ion to electron temperature ratio, $\tau_{i / e}$ as a function of (b) $L_{c}$, (d) PSOL and (f) $n_{u}$.

The plasma parameter $\mathrm{T}_{\mathrm{eu}}, \mathrm{T}_{\mathrm{iu}}, \mathrm{T}_{\mathrm{ed}}, \tau_{\mathrm{i} / \mathrm{e}}$ and $\mathrm{n}_{\mathrm{ed}}$ versus connection length $\mathrm{L}_{\mathrm{c}}$, SOL power $\mathrm{P}_{\mathrm{SOL}}$, and upstream electron density $\mathrm{n}_{\mathrm{u}}$ are calculated and shown in figure 18. As seen in figure 18(a) and (b), by fixing the island size, $\mathrm{P}_{\mathrm{SOL}}$ and $\mathrm{n}_{\mathrm{u}}$, increasing $\mathrm{L}_{\mathrm{c}}$ would almost linearly increase $\mathrm{T}_{\text {eu }}, \mathrm{T}_{\mathrm{iu}}$ and $\mathrm{n}_{\text {ed. }}$. This could be understood straightforward since longer magnetic field lines lead to higher particle and energy confinement time. As a consequence of the momentum and energy balance, $\mathrm{T}_{\mathrm{ed}}$ slightly decreases with the increase of $\mathrm{L}_{\mathrm{c}}$. Since the term of $7 Q_{e q u} L_{c}^{2} / 4 \kappa_{e}$ increases with $\mathrm{L}_{\mathrm{c}}$, $\tau_{i / e}$ (black dot) slightly decreases with increasing the connection length. In the case of fixed $\mathrm{L}_{\mathrm{c}}$ and $n_{u}$, increasing $P_{S O L}$ would also increase $T_{e u}, T_{i u}$, and $T_{e d}$ (seen in figure 18(d)). Due to fixed upstream electron density, the downstream electron density would first increase to conserve the pressure and then saturate to a certain value (seen in figure 18(c)). For the conditions of fixed $\mathrm{L}_{\mathrm{c}}$ and $\mathrm{P}_{\mathrm{SOL}}, \mathrm{T}_{\mathrm{eu}}$ and $\mathrm{T}_{\mathrm{iu}}$ gradually decrease as $\mathrm{n}_{\mathrm{u}}$ increases (seen in figure 18(f)). Since the thermal 
equilibrium term $Q_{e q}$ is proportional to $n_{u}, T_{e u}$ and $T_{i u}$ are close to each other for high $n_{u}$ value. As a consequence, $\tau_{i / e}$ gradually decreases with $\mathrm{n}_{\mathrm{u}}$ and approaches to 1 . In addition, $\mathrm{n}_{\mathrm{ed}}$ is general double of $\mathrm{n}_{\mathrm{u}}$ and gradually increases with $\mathrm{n}_{\mathrm{u}}$.

Comparing figure 18 with figures $9,11,13$ and 16 , the theory results of the $\mathrm{P}_{\mathrm{SOL}}$ and $\mathrm{n}_{\mathrm{u}}$ dependence of plasma parameters are qualitatively consistent with the RFA measurement. While the plasmas parameters dependence on $\mathrm{L}_{\mathrm{c}}$ could not be directly compared with the experiments, since increasing the control coil current not only decreases $L_{c}$ but also shift inward the separatrix by expanding the island size.

\section{Conclusions}

In this work, the ion temperature profile at the plasma boundary has been measured on W7-X during the last experimental campaign OP1.2b by a retarding field analyzer (RFA) probe. The RFA probe head was mounted on the front-end of a MPM located on the low field side of W7-X with toroidal location $\left(\varphi=-159.26^{\circ}\right)$ such that it crosses a magnetic island in the standard divertor configuration. The ion temperature is studied only in the forward side since the modulated ion current measured in the backward side is contaminated by noises. The experiments are limited to high density regime to minimize the flow effect on the ion temperature in single side. The experimental observations show an ion temperature shoulder near the location where a sudden change of the magnetic field connection length appears in the edge island. The edge ion temperature profile has been studied by slightly varying the edge island size by turning the control coil current $\mathrm{I}_{\mathrm{cc}}$, ECRH power $\mathrm{P}_{\mathrm{ECRH}}$ and plasma density. The results show that the $\mathrm{T}_{\mathrm{i}}$ shoulder decreases when the island width is expanded. Since enlarged island size not only shrinks the connection length distribution but also shifts inward the separatrix, it's hard to distinguish these two effects on the ion temperature measurement. The other effects of plasma beta, toroidal current, and magnetic stochastization may also play a role on the edge transport and are currently investigated. However, for the plasma parameters of the RFA experiments, given their rather moderate beta-values $(<2 \%)$ and the small toroidal currents $(<3 \mathrm{kA})$, the effect of increasing the island size is expected to play the major role. Nevertheless, a final conclusion cannot be drawn yet.

For a fixed control coil current, the $\mathrm{T}_{\mathrm{i}}$ shoulder increases gradually with the SOL kinetic power. A decrease of the $T_{i}$ shoulder was also observed when the central line-integrated electron density, $\mathrm{n}_{\mathrm{el}}$, increases from $7 \times 10^{19} \mathrm{~m}^{-2}$ to $9 \times 10^{19} \mathrm{~m}^{-2}$. In addition, the edge ion-to-electron temperature ratio $\tau_{i / e}$ has been obtained by RFA probe. $\tau_{i / e}$ roughly decreases for larger $\mathrm{I}_{\mathrm{cc}}$ and $\mathrm{n}_{\mathrm{el}}$, while increases for larger $\mathrm{P}_{\mathrm{SOL}}$. Furthermore, a normalized ion collisionality $v_{S O L, i}^{*} \equiv L_{c} / \lambda_{i i} \approx 10^{-16} n_{u} L_{c} / T_{i u}^{2}$ is 
introduced to study the correlation between the collisionality and the plasma parameters. The results show that the ion temperature gradually decreases with $v_{S O L, i}^{*}$. Increasing the control coil currents would expand the island geometry, which in general reduce the ion temperature, electron temperature and density. $\tau_{i / e}$ has been found to be gradually decreased with $v_{S O L, i}^{*}$ from $\sim 2$ to $\sim 1$ when the normalized ion collisionality increases from $\sim 10$ to $\sim 200$. This evolution seems independent on the control coil current conditions even though the plasma parameters and island configuration have been changed. The RFA has also measured that the nitrogen seeding from the upper horizontal divertor port could reduce and flatten the edge ion temperature profile. To understand the experimental measurement, a two-point model including ion temperature is introduced, which shows qualitatively consistent with the experimental measurement.

\section{Acknowledgments}

The authors deeply appreciate the continued research and operational efforts of the entire W7-X team. The authors thank to discussing with D. Reiser, M. Rack, M. Dreval, M. Hirsch, A. Dinklage and G. Wurden. This work has been carried out within the framework of the EUROfusion Consortium and has received funding from the Euratom research and training programme 2014-2018 and 2019-2020 under grant agreement No 633053, and was supported by the International Postdoctoral Exchange Fellowship Program Nos. 20170009, the National Natural Science Foundation of China under Grant Nos. 11705237.

\section{Figures and figure captions}

\section{$7 \quad$ References}

[1] Guo H. Y. et al 1996 Contrib. Plasma Phys. 3681

[2] Pitts R. A. et al 2003 Rev. Sci. Instrum. 744644

[3] Kočan M. et al 2008 Rev. Sci. Instrum. 79073502

[4] Brunner D. et al 2013 Rev. Sci. Instrum. 84033502

[5] Nedzelskiy I. S. et al 2006 Rev. Sci. Instrum. $7710 \mathrm{E} 729$

[6] Dreval M. et al 2009 Rev. Sci. Instrum. 80103505

[7] Li Y. L. et al 2016 Rev. Sci. Instrum. 87123503

[8] Ezumi N. et al 2003 J. Nucl. Mater. 313696

[9] Guzmán F. et al 2009 J. Nucl. Mater. 3901127

[10] Strumberger E. 1996 Nucl. Fusion 36891

[11] Renner H. et al 2002 Plasma Phys. Control. Fusion 441005

[12] Pedersen T. S. et al 2016 Nat Commun 713493

[13] Drews P. et al 2017 Nucl. Fusion 57126020

[14] Killer C. et al 2019 Nucl. Fusion 59086013

[15] Feng Y. et al 2006 Nucl. Fusion 46807

[16] Wolf R. C. et al 2017 Nucl. Fusion 57102020

[17] Dinklage A. et al 2018 Nature Phys. 14855

[18] Satheeswaran G. et al 2017 Fusion Eng. Des. 123699

[19] Nicolai D. et al 2017 Fusion Eng. Des. 123960 
[20] Henkel M. et al 2018 Plasma Sci. Technol. 20054001

[21] Valsaque F. et al 2002 Phys. Plasmas 91806

[22] Langmuir I. and Compton K. T. 1931 Rev. Mod. Phys. 3191

[23] Molvik A. W. 1981 Rev. Sci. Instrum. 52704

[24] Brillouin L. 1945 Phys. Rev. 67260

[25] Renner H. et al 2000 Nucl. Fusion 401083

[26] Bozhenkov S. A. et al 2013 Fusion Eng. Des. 882997

[27] Drews P. et al 2019 Nuclear Materials and Energy 19179

[28] Zhang D. et al 2010 Rev. Sci. Instrum. 81 10E134

[29] Kočan M. et al 2008 Plasma Phys. Control. Fusion 50125009

[30] Stangeby P. C. 2000 The plasma boundary of magnetic fusion devices: CRC Press) 